\title{
Digitales Coaching - mehr als ein Trend?
}

\section{Eine vergleichende Analyse des Nutzungsverhaltens digitaler Medien im Coaching bei Coaches und Personalverantwortlichen}

\author{
Thomas Bachmann ${ }^{1}$ (D) J Julia Marie Gramlich ${ }^{2} \cdot$ Henrike Barth $^{3}$
}

Online publiziert: 18. Dezember 2019

(c) Der/die Autor(en) 2019

\section{Zusammenfassung}

Moderne Medien bestimmen das Berufsleben ständig, überall und tiefgreifend. Auch für die Profession des Coachings gibt es bereits zahlreiche digitalisierte Angebote. Trotz vielfach benannter Vorteile existiert bislang nur wenig Forschung zur Digitalisierung im Coaching. Der vorliegende Artikel illustriert anhand zweier empirischer Untersuchungen Hintergründe des Nutzungsverhaltens digitalisierten Coachings bei externen Coaches und Personalverantwortlichen in deutschen Unternehmen. Unter Verwendung der Theorie des geplanten Verhaltens nach Ajzen $(1985,1991)$ zeigt sich bei beiden Berufsgruppen, dass die subjektive Norm in Form von Peers, Organisationen sowie der Gesellschaft den größten Einfluss auf die Intention hat, digitale Angebote und Tools im Coaching zu nutzen.

Schlüsselwörter Coaching · Digitalisierung · Theorie des geplanten Verhaltens

\section{Digital Coaching-more than a Trend?}

A Comparative Analysis Between Coaches and Human Resource Managers Regarding the Usage Behavior of Digital Media in Coaching

\begin{abstract}
Every day, modern media constantly and profoundly determine our professional lives. Concomitant, an array of digital offers already exists for the profession of coaching. Despite multiple advantages being highlighted, so far only little research consists concerning the process of digitalization in coaching. The present article thus illustrates the background of usage behavior regarding digital coaching of coaches and human resource managers in German companies by means of two empirical studies. Using the Theory of Planned Behavior (Ajzen, 1985; 1991; 2005) it becomes apparent that the social norm in form of other colleagues, organizations as well as the society as a whole exerts the greatest influence on the intention using digital media in coaching of both coaches and human resource managers.
\end{abstract}

Keywords Coaching $\cdot$ Digitalization · Theory of Planned Behavior

\footnotetext{
Thomas Bachmann

bachmann@artop.de

Julia Marie Gramlich

julia.gramlich@unisg.ch

Henrike Barth

henrikebarth@outlook.de
}

\footnotetext{
1 artop $\mathrm{GmbH}$, Institut an der Humboldt-Universität zu Berlin, Christburger Str. 4, 10405 Berlin, Deutschland

2 Universität St. Gallen, Girtannerstr. 6, 9000 St. Gallen, Schweiz

3 Zwinglistraße 8, 10555 Berlin, Deutschland
} 


\section{Einleitung}

Über 50 Jahre nachdem Joseph Weizenbaum (1966) den ersten digitalen Psychotherapeuten ELIZA entwickelte, hat die digitale Revolution heute viele andere Beratungsberufe durchdrungen. So auch die Profession des Coachings: Avatare, Video-Chats und digitale Whiteboards stehen programmatisch für die zunehmenden, digitalen Veränderungen im ,populärsten Personalentwicklungsinstrument“ (Deutscher Bundesverband Coaching 2013, S. 6). Welche Auswirkungen jedoch hat die Digitalisierung für das Coaching? Auf der einen Seite betonen Kritiker, dass das Coaching vom Zwischenmenschlichen lebt und deshalb nicht durch digitale Medien ersetzt werden kann (Heller et al. 2018). Demgegenüber wird Coaching-Praktizierenden neben „Ignoranz" und „Skepsis“ unter anderem vorgeworfen, sie ,blieben in den Rillen alter Gewohnheiten verhaftet" (Geißler 2018, S. 30). Wieder andere befürchten, dass digitale Beratungssettings eine Zunahme an unqualifizierter Konkurrenz auf einem immer unkontrollierbarer werdenden Markt bedeuten (Fietze 2016).

Und wie steht es um die Meinung derer, die Coaching als Personalentwicklungsinstrument in Organisationen anbieten und implementieren? Da Coaching zukünftig an Bedeutung gewinnen und sich speziell nach der Begleitung von Change-Prozessen zum wichtigsten Personalentwicklungsinstrument ausbilden wird (Schermuly et al. 2012), rückt neben der Meinung von Coaches auch die Frage, wie Personalverantwortliche in Organisationen den Einsatz digitaler Tools im Coaching bewerten, verstärkt in den Vordergrund. Welche Rolle spielen hierbei z.B. die Sorge um Datenschutz, unterschiedliche Verständnisse von Coaching oder die Meinung anderer Kolleg/innen bzw. Peers?

Der vorliegende Artikel illustriert anhand zweier empirischer Untersuchungen verschiedene Hintergründe des Nutzungsverhaltens digitalisierten Coachings sowohl bei professionellen Coaches als auch bei Personalverantwortlichen in deutschen Unternehmen. Beide Gruppen sind beim Coaching im Kontext von Organisationen in ihrem Wirken eng miteinander verknüpft. Personalverantwortliche prägen durch ihre Art der Implementierung und Nutzung bzw. des Angebots von Coaching das Coachingverständnis der Organisationen (Bachmann 2016). Sie wählen externe Coaches aus und beauftragen sie und nehmen damit als Auftraggebende auch Einfluss auf deren Handeln und Leistungserbringung. Weiterhin haben Personalverantwortliche neben dieser „Vermittlungsaufgabe“ auch noch eine Beratungsfunktion für potentielle Nachfragende bzw. Auftraggebende aus der Organisation, indem sie Vorgespräche führen und möglichen Coachingbedarf identifizieren. Nicht selten haben sie darüber hinaus auch die Funktion von internen Coaches. Für eine Untersuchung der Digitalisierung im Coaching ist daher ein Vergleich von externen Coaches und Personalverantwortlichen nicht nur interessant, sondern erlaubt auch Rückschlüsse auf Ursachen für Entwicklungstrends im Coachingfeld und speziell innerhalb der Profession Coaching.

\section{Theoretischer Hintergrund}

Was genau bedeutet digitalisiertes Coaching? Dieser Frage wird zunächst anhand einer differenzierten Darstellung der Digitalisierung im Coaching digitalisierten Coachings nachgegangen. Anschließend wird mithilfe der Theorie des geplanten Verhaltens von Ajzen $(1985 ; 1991)$ erklärt, welche Faktoren die Entscheidung von Coaches und Personalverantwortlichen beeinflussen, digitale Tools im Coaching tatsächlich einzusetzen.

\subsection{Digitalisierung von Coaching}

In Anlehnung an Bachmann und Fietze (2018) und Pascal et al. (2015) stellt Abb. 1 die Bereiche dar, in denen sich die Digitalisierung von Coaching vollzieht bzw. zu erwarten ist. Diese werden im Folgenden kurz erläutert.

$\mathrm{Zu}$ Beginn eines Coachingprozesses steht die Auswahl eines geeigneten Coachs (Bachmann und Fietze 2018; Pascal et al. 2015). Im organisationalen Kontext wird grundsätzlich zwischen den organisationsexternen und den organisationsinternen Coaches unterschieden, wobei Coaching in der Praxis am häufigsten mit ersteren aufgrund ihrer betriebsfremden, unvoreingenommenen Rolle durchgeführt wird (Rauen 2003; Schwaemmle und Staehelin 2002). Plattformen wie coachdatenbank.de, xing.com, coachhub.com oder sharpist.com erleichtern die Suche nach Coaches und ermöglichen außerdem online Kontaktaufnahmen sowie Zugang zu detaillierteren Coach-Profilen und zum Teil auch zu Bewertungen der Coaches. Die Digitalisierung dieses dem eigentlichen Coachingprozess vorgeschalteten Bereichs der „Coach-Auswahl“ (Pascal et al. 2015, S. 103) oder des „Coaching-Marktes“ (Bachmann und Fietze 2018)

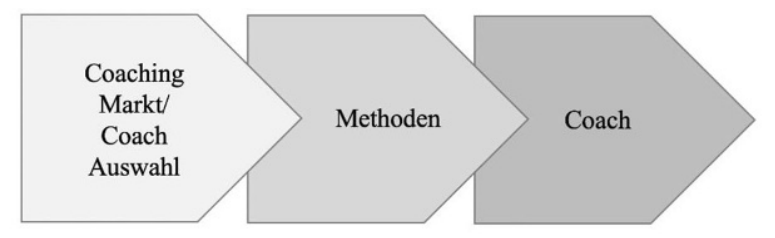

Kommunikation

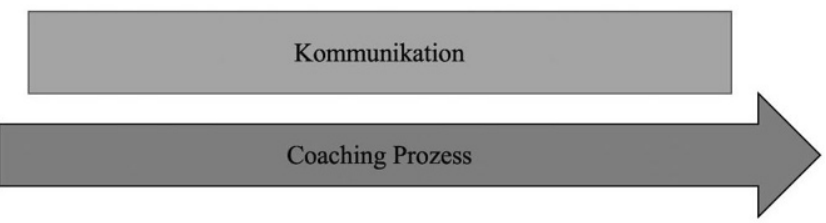

Abb. 1 Bereiche der Digitalisierung von Coaching 
eröffnet so Möglichkeiten einer effizienten, kriteriengeleiteten und ortsungebundenen Coach-Suche. Pascal et al. (2015) antizipieren für diesen Bereich z.B. die Anwendung von Algorithmen, um eine ideale Passung zwischen Coach und Klienten zu gewährleisten.

Neben der Coach-Auswahl können auch klassische Coaching-Methoden wie die Systemaufstellung digitalisiert durchgeführt werden. Ein Beispiel hierfür ist das 3D-visuelle E-Coaching Tool proreal.world, das mit Avataren sowie diversen Symbolen und Landschaften auf eine digitale Aufstellungsarbeit ausgerichtet ist. Ein weiteres Beispiel für den Bereich digitalisierter coachingübergreifender Methoden bietet die Internetseite zoom.us mit Webinarfunktion z.B. über kollaborative Online-Whiteboards, auf denen besprochene Inhalte im Coaching zwischen Coach und Klienten visualisiert werden können.

Nicht zuletzt ist die Digitalisierung auch in der Instanz des Coachs selbst angekommen. So kann dieser durch Selbstcoaching-Formate wie digitale Diagnostik, OnlineVideos und Online-Arbeitsaufgaben im Rahmen von festen Modulen und Themenblöcken teilweise ersetzt werden. Beispiele für das Konzept der geleiteten Selbsthilfe sind e-selfcoaching.ch und coach.me, im Therapiekontext selfapy.de oder die App ada.com zur Selbst-Diagnostik im medizinischen Krankheitsfall. Alle Entwicklungen zielen darauf $a b$, zukünftig die Instanz des Coaches der anderer Helferpersonen mithilfe von Algorithmen und/oder künstlicher Intelligenz, manchmal auch repräsentiert durch Avartare, zu ersetzen.

Als Umrahmung der bereits beschriebenen Formen digitalisierten Coachings kann auch der Coachingprozess an sich digitalisiert ausgelegt werden. So bieten Softwareapplikationen wie coachaccountable.com und coachingcloud.com digitalisierte Terminkoordinationen, Dokumentationen, Erinnerungen sowie Rechnungsstellungen und Evaluationen des Coachingerfolgs an (Bachmann und Fietze 2018). Aufgrund der Schwierigkeit, Coaching generell zu evaluieren (Carter 2006), werden bisher lediglich digitalisierte Fragebögen wie z.B. surveymonkey.co.uk eingesetzt. Das Center for Creative Leadership (CLL) entwickelte ein Evaluationstool, das Verhaltensveränderungen und Erfolge der Klient/innen mittels laufend aktualisierter Beobachtungsdaten verschiedener Stakeholder wie Coach und Führungskraft messen soll (Pascal et al. 2015). Durch ein hohes Standardisierungsmaß der Daten können Organisationen so zukünftig auf einen Blick Erfolge von Coaches und Coaching-Programmen ermitteln. Die Vergleichbarkeit von Coaching-Daten ist gerade für den Arbeitsbereich von Personalverantwortlichen hinsichtlich Coaching-Angebot und Coach-Auswahl relevant.

Schließlich lässt sich auch die allen zuvor genannten Aspekten zugrundeliegende Kommunikation zwischen Coach und Klient/innen digitalisieren. Diese Form des Coa- chings über digitale Kanäle wie z.B. Skype oder E-Mail ist die bereits am längsten etablierte Form digitalisierten Coachings (Bachmann und Fietze 2018), wobei die Professionalität dieser Vorgehen aus Datenschutzgründen kritisch diskutiert werden müssen. Internetseiten wie cai-world.com und gotomeeting.com bieten darüber hinaus weitere Kommunikations-Tools wie beispielsweise Desktop Sharing, Chaträume und Sitzungsaufzeichnungen an.

Unter digitalisiertem Coaching verstehen wir also zusammenfassend, wenn einer der fünf beschriebenen Bereiche des Coachingsprozesses durch digitale Plattformen oder Tools substituiert oder unterstützt wird.

\subsection{Theorie des geplanten Verhaltens}

Menschliches Verhalten zu erklären, stellt seit jeher eine große Herausforderung psychologischer Forschungen dar. Die Theorie des geplanten Verhaltens von Ajzen (1985; 1991) unternimmt genau diesen Versuch und dient den vorliegenden Untersuchungen als Erklärungshilfe für das spezifische Verhalten von Coaches und Personalverantwortlichen, digitalisierte Formen von Coaching zu nutzen.

Ajzen (1985, S. 11) beschreibt Verhalten als „following along lines of more or less well-formulated plans“. Erst diese implizit oder explizit formulierten Pläne ermöglichen das Erreichen von Verhaltenszielen und werden von $\mathrm{Aj}$ zen als Intentionen bezeichnet. Jedoch münden nicht alle Intentionen zwangsläufig in tatsächlichem Verhalten. So können z.B. äußere Umstände verhindern, dass ein spezifisches Verhalten überhaupt gezeigt werden kann: Herr Klöbner möchte seiner Nachbarin selbstgebackene Kekse vorbeibringen, diese ist jedoch nicht zu Hause, sodass die intendierte Handlung gar nicht erst stattfinden kann. Daher definiert Ajzen Intentionen dahingehend, dass sie den Versuch, spezifisches Verhalten zu zeigen, vorhersagen können, nicht jedoch die Handlung selbst. Weiterhin können Intentionen selbst situationsspezifisch angepasst, modifiziert oder gar fallen gelassen werden: Herr Waldvogel möchte z.B. unbedingt $15 \mathrm{~kg}$ abnehmen, lockert diese Intention jedoch auf der Hochzeit seiner Nichte erheblich, als er das üppige Speisebuffet erblickt. Ein zunehmender Zeitabstand zwischen Intention und Situation, in der das spezifische Verhalten gezeigt werden soll, vergrößert hierbei die Wahrscheinlichkeit, dass unvorhergesehene Ereignisse eben jene Intention verändern können. Nach Ajzen (1985) lassen sich Veränderungen in Intentionen durch die drei Determinanten:
1. Einstellung zum Verhalten (kurz: Einstellung),
2. subjektive Norm und
3. die bereits erwähnte wahrgenommene Verhaltenskontrol- le 


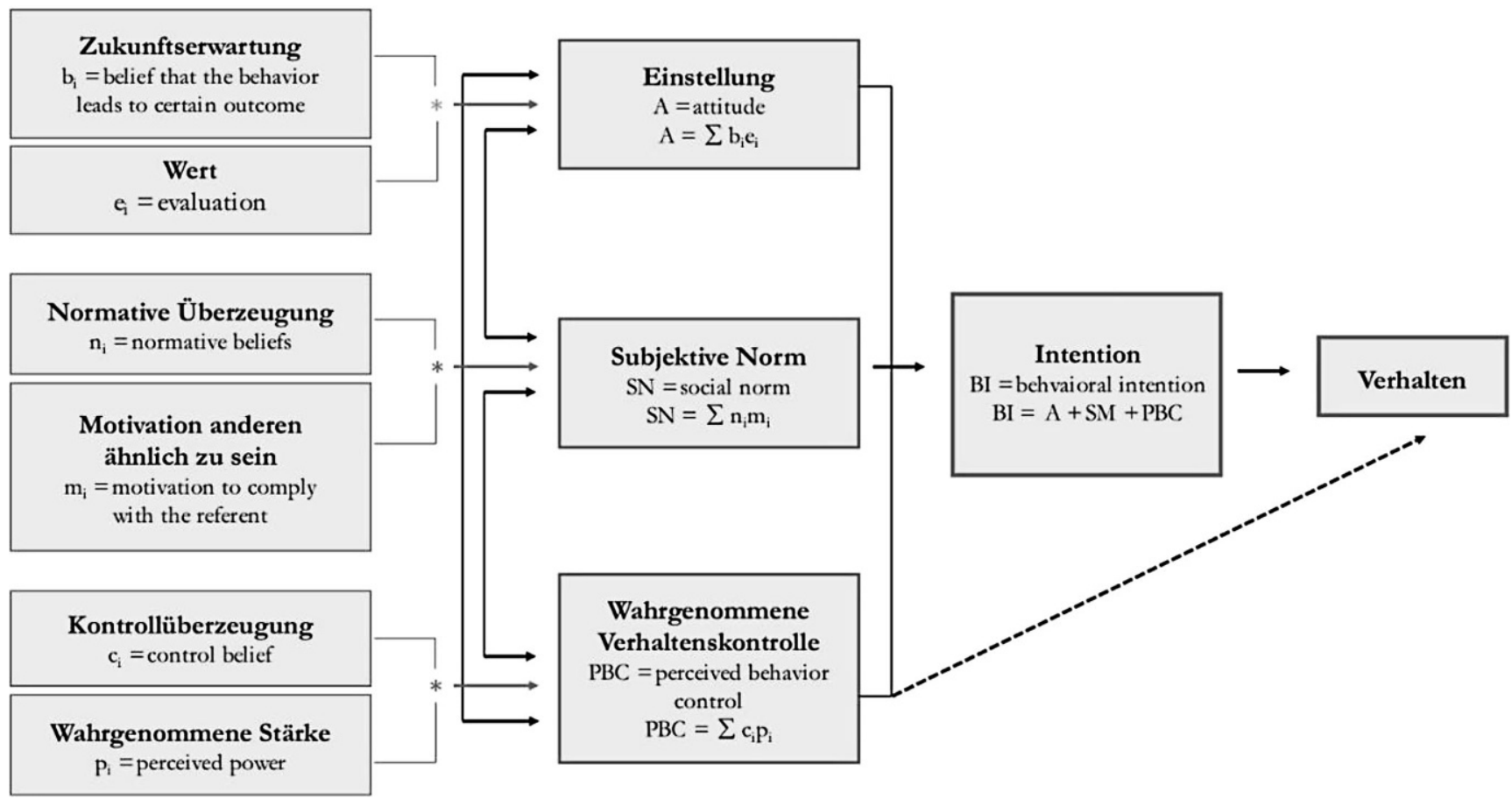

Abb. 2 Theorie des geplanten Verhaltens. (Eigene Darstellung in Anlehnung an Wiethoff 2004 und Ajzen 2005)

erklären. Diese drei Determinanten werden im Folgenden einzeln beleuchtet. Abb. 2 veranschaulicht ergänzend die Komponenten der Theorie des geplanten Verhaltens sowie deren jeweilige Beziehungen zueinander.

1. Ajzen (1985) bezeichnet Einstellungen als Verhaltensüberzeugungen, also als eine Art persönlicher Bewertung von Verhalten. Dabei beziehen sich Einstellungen im Rahmen der Theorie des geplanten Verhaltens lediglich auf das Verhalten an sich, nicht jedoch auf Personen oder Objekte. Nach Ajzen (1985, S. 13) setzen sich Einstellungen aus der Bewertung einer Person hinsichtlich des Ergebnisses einer Handlung sowie der Stärke dieser Bewertung zusammen. Wie in Abb. 2 dargestellt, sind Einstellungen (A; attitude) dabei proportional zum summativen Index bestehend aus der Multiplikation von Zukunftserwartung ( $b$; belief that the behavior leads to a certain outcome) und Wert (e; evaluation of outcomes).

2. Nach Ajzen (1985) stellt die subjektive Norm die individuelle Wahrnehmung des sozialen Drucks dar, ein spezifisches Verhalten zeigen oder nicht zeigen zu müssen. So kann die subjektive Norm auch als eine normative Überzeugung verstanden werden, die sich an der spezifischen Meinung einer individuellen Referenzgruppe orientiert. Wie in Abb. 2 dargestellt, setzt sich die subjektive Norm (SN; social norm) hierbei aus der multiplikativen Verknüpfung von normativen Überzeugungen ( $n$; normative beliefs) und der Motivation, einer spezifischen Refe- renzgruppe möglichst ähnlich $\mathrm{zu}$ sein $(\mathrm{m}$; motivation to comply with the referent) zusammen (Ajzen 1991).

3. Die wahrgenommene Verhaltenskontrolle stellt nach Ajzen (1991) die subjektiv empfundene Einfachheit oder Schwierigkeit dar, ein spezifisches Verhalten zeigen zu können. Diese individuelle Einschätzung wird maßgeblich durch vorhandene oder nicht vorhandene Ressourcen und Handlungsmöglichkeiten beeinflusst. Weiterhin bedingen eigens gemachte Erfahrungen aus der Vergangenheit, Erfahrungen von näherstehenden Personen wie Familie oder Freund/innen sowie weitere Informationsquellen die individuell wahrgenommene Verhaltenskontrolle von Personen. Wie in Abb. 2 dargestellt, setzt sich die wahrgenommene Verhaltenskontrolle ( $P B C$; perceived behavior control) dabei multiplikativ aus der situationsspezifischen Kontrollüberzeugung ( $c$; control belief) sowie deren empfundene Stärke ( $p$; perceived power) zusammen. Zudem kann die wahrgenommene Verhaltenskontrolle unabhängig von der Intention als einzige Determinante einen direkten Einfluss auf das Verhalten haben.

Nach Ajzen (1991) kann die Gewichtung der drei Determinanten zwischen spezifischen Verhaltensweisen und Situationen variieren: So kann z. B. bei einer schwachen Einstellung die subjektive Norm eine starke Vorhersagekraft für ein spezifisches Verhalten haben. Darüber hinaus formuliert Ajzen, dass nur Intentionen einen direkten Einfluss auf Verhalten haben, Persönlichkeitseigenschaften und demographische Variablen hingegen nicht. Für die vorlie- 
gende Studie folgt daraus, dass die Einstellung gegenüber der Nutzung digitaler Formen von Coaching, die subjektive Norm einer relevanten Referenzgruppe und schließlich die wahrgenommene Verhaltenskontrolle die Intention der Nutzung digitalisierten Coachings von Personalverantwortlichen determinieren.

Verschiedene Metaanalysen (z.B. Ajzen 1991; Armitage und Conner 2001; Hausenblas et al. 1997; Randall und Wolff 1994) haben die einzelnen Determinanten der Theorie des geplanten Verhaltens empirisch überprüft. So kommen Hausenblas et al. (1997) z.B. zu dem Ergebnis, dass die Korrelationen der einzelnen Determinanten mit der Intention $\left(r_{\mathrm{A}}=0,52 ; r_{\mathrm{SN}}=0,27 ; r_{\mathrm{PBC}}=0,43\right)$ insgesamt höher ausfallen als mit dem Verhalten $\left(r_{\mathrm{A}}=0,39 ; r_{\mathrm{SN}}=0,09 ; r_{\mathrm{PBC}}=0,45\right)$, was als empirischer Beleg für die Variable der Intention gelten kann. Armitage und Conner (2001) berechnen sogar eine signifikante multiple Korrelation der drei Determinanten mit der Intention von $r=0,57$. Die Betrachtung der Stärke des Einflusses der einzelnen Determinanten auf die Intention zeigt, dass sich die Einstellung als stärkster, die subjektive Norm als schwächster Prädiktor herausstellt. Dieses Ergebnis deckt sich mit anderen empirischen Studienergebnissen (z. B. Ajzen 2005).

Bezüglich des Zusammenhangs zwischen der Intention und dem Verhalten (s. Abb. 2) ermitteln Randall und Wolff (1994) eine Korrelation von $r=0,45$. Armitage und Conner (2001) kommen hier zu einem ähnlichen Ergebnis von $r=47$. Durch Hinzunahme der Variable wahrgenommene Verhaltenskontrolle berechnen sie sogar eine multiple Korrelation von $R=0,52$. So können mit der Theorie des geplanten Verhaltens neben der Intention auch Vorhersagen über tatsächliches Verhaltens gemacht werden: Nach Armitage und Conner (ebd.) klärt die Theorie des geplanten Verhaltens insgesamt 20\% der Varianz in Messungen zukünftigen Verhaltens auf und kann damit als valides Erklärungsmodell für die vorliegende Studie gelten.

\section{Fragestellungen und Hypothesen}

Das Ziel der hier vorgestellten Studie ist es, anhand der folgenden zwei Fragestellungen Aufschluss über die Hintergründe sowie Unterschiede des Nutzungsverhaltens digitalisierten Coachings bei Coaches und Personalverantwortlichen zu geben.

Fragestellung 1 Welche Formen digitalisierten Coachings nutzen externe Coaches und Personalverantwortliche bereits, bzw. können sich vorstellen, in Zukunft zu nutzen?

In Abschn. 2.1 wurden fünf Bereiche digitalisierten Coachings in Anlehnung an Bachmann und Fietze (2018) und Pascal et al. (2015) vorgestellt: Coaching-Markt bzw. -Auswahl, Methoden, Coach, Kommunikation und Coaching-
Prozess. Aufgrund mangelnder empirischer Untersuchungsergebnisse für eine wissenschaftlich fundierte Formulierung von Hypothesen wird hier ein exploratives Vorgehen gewählt.

Fragestellung 2 Inwiefern beeinflussen Einstellungen, subjektive Norm und wahrgenommene Verhaltenskontrolle von externen Coaches und Personalverantwortlichen die Nutzungsintention und das Nutzungsverhalten von digitalen Tools im Coaching?

In Abschn. 2.2 wurde erläutert, warum die Theorie des geplanten Verhaltens der vorliegenden Untersuchung als theoretisches Erklärungsmodell zur Vorhersage willentlichen Verhaltens zugrunde liegt. Die drei Determinanten Einstellung, subjektive Norm und wahrgenommene Verhaltenskontrolle beeinflussen dabei die Intention, spezifisches Verhalten zu zeigen. Dabei variiert die Stärke der Beeinflussung der Determinanten situationsspezifisch (Ajzen 1991; Hausenblas et al. 1997; Armitage und Conner 2001). Demgemäß lassen sich zur Beantwortung der zweiten Forschungsfrage folgende Hypothesen formulieren:

Hypothese $\mathrm{H}_{1}$ Es besteht ein positiver Zusammenhang zwischen der Einstellung von Coaches und Personalverantwortlichen bezüglich digitaler Plattformen und Tools für Coaching und ihrer Intention, diese im Coaching zu nutzen.

Hypothese $\mathrm{H}_{2}$ Es besteht ein positiver Zusammenhang zwischen der subjektiven Norm der Coaches und der Personalverantwortlichen hinsichtlich digitaler Plattformen und Tools für Coaching und ihrer Intention, diese im Coaching zu nutzen.

Hypothese $\mathrm{H}_{3}$ Es besteht ein positiver Zusammenhang zwischen der wahrgenommenen Verhaltenskontrolle von Coaches und Personalverantwortlichen in Bezug auf die Nutzung digitaler Plattformen und Tools für Coaching und ihrer Intention, diese im Coaching zu nutzen.

Hypothese $\mathrm{H}_{4}$ Es besteht ein positiver Zusammenhang zwischen der Intention von Coaches und Personalverantwortlichen, digitale Plattformen und Tools für Coaching zu nutzen, und ihrem tatsächlich gezeigten Nutzungsverhalten.

Fragestellung 3 Welche Unterschiede gibt es zwischen Einstellungen, subjektiver Norm und wahrgenommener Verhaltenskontrolle sowie Intention und Verhalten zwischen externen Coaches und Personalverantwortlichen hinsichtlich der Nutzung digitaler Plattformen und Tools im Coaching?

Aufgrund ihrer Zugehörigkeit zu Organisationen kann davon ausgegangen werden, dass Personalverantwortliche eher als „Treibende“ für neue Technologien wirken, da sie dem Effizienz- und Innovationsdruck ihrer Organisation un- 
terliegen. Damit kann angenommen werden, dass sie somit Einfluss auf das Nutzungsverhalten digitaler Plattformen und Tools der Coaches ausüben.

Hypothese $\mathrm{H}_{5}$ Personalverantwortliche sind im Vergleich zu externen Coaches eher nutzungsaffin bezügliche digitaler Plattformen und Tools im Coaching, was sich in positiveren Einstellungen, einem stärkeren Einfluss der sozialen Norm und einer höher ausgeprägten Intention ausdrückt.

\section{Methode}

\subsection{Stichprobe und Studiendesign}

Als Basis für die vorliegende empirische Untersuchung dient eine Stichprobe von 102 Coaches und 91 Personalverantwortlichen in Deutschland. Zur Beantwortung der aufgeführten Forschungsfragen wurden zwei unabhängige quantitative Online-Befragungen durchgeführt. Die Erhebungen fanden im Frühjahr bis Sommer 2018 statt. Für die Datenerhebung der Coaches wurden 700 Coaches der Rauen Coaching-Datenbank persönlich per E-Mail kontaktiert. Darüber hinaus wurden der Verteiler des Coaching-Ausbildungsinstituts artop - Institut an der Humboldt-Universität zu Berlin, persönliche Kontakte und die Xing-Gruppe der International Coaching Federation zur Gewinnung von Teilnehmern verwendet. Die teilnehmenden Personalverantwortlichen wurden über den Verteiler von artop, über XING.com, LinkedIn.com und den Bundesverband Deutscher Personalmanager (BDP) sowie über persönliche Kontakte akquiriert. Da in Deutschland kein System existiert, in dem sich alle Personalverantwortlichen und Coaches registrieren lassen müssen, ist die Generierung einer $\mathrm{Zu}$ fallsstichprobe durch Listenauswahl unmöglich (Diekmann 2017, S. 381).

Die teilnehmenden Coaches waren im Mittel 54 Jahre alt, darunter $48 \%$ männlichen und $52 \%$ weiblichen $\mathrm{Ge}-$ schlechts. Ein Großteil der Befragten gab als höchsten Bildungsabschluss Hochschule oder Fachhochschule (71\%) an. Weitere $18 \%$ der Coaches tragen einen Doktortitel. Am häufigsten gaben die Teilnehmenden eine Ausbildung in den Bereichen Geisteswissenschaften (38\%; Mehrfachnennungen waren möglich), Wirtschaft (34\%), Pädagogik $(14 \%)$ und dem sozialen Bereich $(11 \%)$ an. Die Berufserfahrung gaben $65 \%$ der Coaches mit mehr als 10 Jahren an, gefolgt von 17\% mit 5-10 Jahren, 6\% mit 3-5 Jahren, 9\% mit 1-3 Jahren und 4\% mit 0-1 Jahr. $67 \%$ der Teilnehmenden arbeiten zu weniger als $50 \%$ für Selbstzahler. Tab. 1 zeigt die prozentualen Häufigkeiten der Themen innerhalb der Coaching Sitzungen der externen Coaches.

Bei der Stichprobe der Personalverantwortlichen beträgt der mittlere Altersdurchschnitt der Stichprobe 43,2 Jah-
Tab. 1 Prozentuale Häufigkeit von Coachingthemen bei externen Coaches (Mehrfachauswahl, $N=102$ )

\begin{tabular}{ll}
\hline $\begin{array}{l}\text { Häufigkeit der Coachingthemen nach Angabe der externen Coaches } \\
\text { (in \%) }\end{array}$ \\
\hline Führung- und Management & 79,4 \\
Karriere/berufliche Entwicklung & 58,8 \\
Persönlichkeitsentwicklung & 57,8 \\
(Team-)Konflikte & 38,2 \\
Standing/persönliches Auftreten & 31,4 \\
Work-Life-Balance & 28,4 \\
Mikropolitik/Entscheidungen & 14,7 \\
\hline
\end{tabular}

Tab. 2 Prozentuale Häufigkeit von Coachingthemen bei Personalverantwortlichen (Mehrfachauswahl, $N=85$ )

Häufigkeit der Coachingthemen nach Angabe der Personalverantwortlichen (in \%)

\begin{tabular}{ll}
\hline Führung- und Management & 75,8 \\
(Team-)Konflikte & 50,4 \\
Persönlichkeitsentwicklung & 46,2 \\
Karriere/berufliche Entwicklung & 37,4 \\
Standing/Persönliches Auftreten & 28,6 \\
Work-Life-Balance & 15,4 \\
Mikropolitik/Entscheidungen & 9,9 \\
\hline
\end{tabular}

re, mehr als die Hälfte der Teilnehmenden waren Frauen $(57,6 \%)$. Als höchsten Bildungsabschluss gaben $78 \%$ der Teilnehmenden einen Fach- oder Hochschulabschluss an. Weitere $13 \%$ sind promoviert. Am häufigsten gaben die Teilnehmenden eine Ausbildung in den Bereichen Wirtschaft $(41 \%)$ und Geisteswissenschaften $(22 \%)$ an. Hinsichtlich der beruflichen Daten der Teilnehmenden gaben $52 \%$ an, mehr als zehn Jahre Berufserfahrung zu besitzen, gefolgt von $18 \%$ mit 6-10 Jahren, 13\% mit 4-5 Jahren, 9\% mit 2-3 Jahren und 7\% mit 0-1 Jahr. 55\% der Personalverantwortlichen haben eine Position als Führungskraft inne. Am häufigsten wurden die Branchen Dienstleistungen und sonstige Branchen (21\%) angegeben. Weitere 14\% der Teilnehmenden arbeiten in der Produktionsbranche. Tab. 2 zeigt die prozentualen Häufigkeiten der Themen aus Sicht der Personalverantwortlichen.

Signifikante Unterschiede zwischen den Coachingthemen sind bei „Karriere“ und bei „Work-Life-Balance“ festzustellen $\left(\chi^{2}=8,87 ; p=0,002\right.$ und $\left.\chi^{2}=4,73 ; p=0,020\right)$. Bei den anderen Themen gibt es hinsichtlich der Häufigkeit eine große Ähnlichkeit, was dafür spricht, dass externe Coaches und Personalverantwortliche hier in vergleichbaren Feldern aktiv sind. Die Häufigere Nennung von Karriere- und WorkLife-Balance-Themen könnte sich daraus ergeben, dass diese bei externen Coaches mehr von Privatzahlenden nachgefragt werden. 


\subsection{Fragebogen}

Zur Beantwortung der Forschungsfragen wurde ein deduktives Vorgehen gewählt, die eingesetzten Fragebögen umfassen je 81 Items. Bis auf die soziodemographischen, beruflichen sowie organisationalen Daten wurde zur Beantwortung der einzelnen Items eine 6-stufige Likert-Skala mit den jeweiligen Polen $1=$ trifft überhaupt nicht $z$ u/nie/gar nicht wichtig bis $6=$ trifft voll zu/immer/sehr wichtig vorgegeben. Zu Beginn sollten die Teilnehmenden ihr Alter, Geschlecht, höchsten Bildungsabschluss, Fachrichtung, Berufserfahrung sowie die drei häufigsten Coaching-Themen angeben.

Für die Theorie des geplanten Verhaltens wurden die einzelnen Variablen wie folgt operationalisiert: Die Determinante Einstellung ergibt sich aus den Variablen Zukunftserwartung $\times$ Wert. Entsprechend wurden für die Variable $\mathrm{Zu}$ kunftserwartung acht Items wie z. B. „Durch die Digitalisierung kann Coaching zukünftig räumlich flexibler werden“ formuliert. Weiterhin wurde analog in acht darauffolgenden Items die Wertung dieser Zukunftserwartungen durch Items wie „Im Zusammenhang mit der Digitalisierung ist mir die räumliche Flexibilität wichtig" abgefragt.

Das Augenmerk bei der Operationalisierung der zweiten Determinante subjektive Norm lag auf den von Wiethoff (2004) formulierten Aspekten der Überzeugung, dass relevante Bezugspersonen der Meinung sind, dass ein spezifisches Verhalten gezeigt werden sollte sowie der Motivation, diesen Bezugspersonen ähnlich zu sein. Ein beispielhaftes Item für den ersten Aspekt ist „Positive Erfahrungen anderer Personalverantwortlicher mit digitalen Medien, Apps und Plattformen im Coaching lösen bei mir Interesse und Neugier aus“. Items des zweiten Aspekts, die Motivation, Bezugspersonen ähnlich zu sein, wurden aufgrund von Reaktanzreaktionen der Proband/innen im Pretest vom endgültigen Fragebogen ausgeschlossen.

Die dritte Determinante, die wahrgenommene Verhaltenskontrolle, setzt sich aus der Kontrollüberzeugung mit beispielhaften Items wie „Meine Organisation bzw. mein Unternehmen verfügt über ausreichend finanzielle Ressourcen, um digitale Coaching-Formate zu verwenden" sowie der wahrgenommenen Stärke zusammen. Ähnlich wie bei der subjektiven Norm wurden die formulierten Items für die wahrgenommene Stärke aufgrund von Reaktanzreaktionen der Probanden im Pretest vom Fragebogen ausgeschlossen.

Schließlich wurden die Variablen Nutzungsintention sowie Nutzungsverhalten durch die in Abschn. 2.1 vorgestellten fünf Bereiche digitalisierten Coachings operationalisiert, wobei bei der Stichprobe der Coaches anstelle der Digitalisierung der Instanz des Coachs selbst die Digitalisierung der Evaluierung gewählt wurde, da bei ersterem der Beruf von Coaches gänzlich überflüssig würde. Für das Nutzungsverhalten wurden für die jeweiligen fünf Berei- che unterschiedliche Items wie z.B. „Für den Einsatz von Coaching in meiner Organisation bzw. meinem Unternehmen nutze ich bereits Online-Plattformen, um Coaches zu finden" formuliert. Dieselben Items wurden analog für die Nutzungsintention mit der veränderten Formulierung „Für den Einsatz von Coaching in meiner Organisation bzw. meinem Unternehmen kann ich mir zukünftig vorstellen, folgende digitale Angebote zu nutzen ..." erstellt.

\subsection{Skalenbildung und Auswertung}

Zur Beantwortung der ersten Forschungsfrage wurden zunächst die Skalen Intention und Verhalten durch den Mittelwert der zugehörigen Items gebildet. Diese wurden anschließend auf ihre internen Konsistenzen überprüft (Bortz 2013, S. 429f). Daraufhin wurden deskriptive Statistiken dieser Skalen vorgenommen.

Für die Berechnung der Hypothesen der zweiten Forschungsfrage wurden ebenso zunächst die Skalen Einstellung, subjektive Norm und wahrgenommene Verhaltenskontrolle durch den Mittelwert der entsprechenden Items gebildet. Im Anschluss daran wurden auch hier die internen Konsistenzen ermittelt. Zur Beantwortung der Hypothesen $\mathrm{H}_{1}, \mathrm{H}_{1}$ und $\mathrm{H}_{3}$ wurden multiple lineare Regression mit den unabhängigen Variablen (UV) Einstellung, subjektive Norm und wahrgenommene Verhaltenskontrolle sowie der Intention bzw. Verhalten als abhängige Variablen (AV) einmal für die Coaches und einmal für die Personalverantwortlichen durchgeführt. Um Gemeinsamkeiten bzw. Unterschiede im Nutzungsverhalten zwischen Coaches und Personalverantwortlichen darzustellen, wurden Mittelwertvergleiche berechnet. Für alle statistischen Tests wurde eine Irrtumswahrscheinlichkeit von $5 \%$ festgelegt.

\section{Ergebnisse}

Zur Beantwortung der ersten Forschungsfrage nach dem aktuellen Nutzungsverhalten sowie der Nutzungsintention digitalen Coachings von Personalverantwortlichen und externen Coaches wurde eine deskriptive Datenanalyse der Häufigkeiten durchgeführt. Zunächst wurden die internen Konsistenzen der verwendeten Skalen durch die Berechnung von Cronbachs Alpha bestimmt (Bortz 2013). Die Skalenparameter sind in Tab. 3 dargestellt. Insgesamt können die internen Konsistenzen der gebildeten Skalen als sehr zufriedenstellen bezeichnet werden, was damit für eine hohe Aussagekraft der Ergebnisse spricht.

Die Mittelwerte liegen bei den Personalverantwortlichen bei den kognitiven und motivationalen Skalen höher als bei den externen Coaches, während die Skalen „Kontrolle“ und „Verhalten“, die sich auf konkretes Tun beziehen, geringer ausgeprägt sind. Ein Mittelwertvergleich der beiden 
Tab. 3 Parameter der verwendeten Skalen

\begin{tabular}{lllll}
\hline \multirow{2}{*}{ Skala } & \multicolumn{2}{l}{ Externe Coaches } & \multicolumn{2}{l}{ Personalverantwortliche } \\
\cline { 2 - 3 } Einstellung & Mean & Cronbachs $\alpha$ & Mean & Cronbachs $\alpha$ \\
Soziale Norm & 3,93 & 0,83 & 4,11 & 0,84 \\
Verhaltenskontrolle & 3,26 & 0,81 & 4,04 & 0,84 \\
Intention & 3,93 & 0,88 & 3,87 & 0,72 \\
Verhalten & 3,95 & 0,91 & 4,30 & 0,92 \\
& 2,81 & 0,88 & 2,57 & 0,89 \\
\hline
\end{tabular}

Stichproben zeigt signifikante Unterschiede bei der sozialen Norm $(F(1,191)=30,19 ; p=0,000)$ und bei der Intention $(F(1,191)=4,38 ; p=0,038)$. Damit zeigt sich, dass die Personalverantwortlichen eher die Digitalisierung im Coaching befördern möchten als die Coaches und sich dabei vor allem stärker an der sozialen Norm orientieren.

Abb. 3 veranschaulicht das Antwortverhalten der beiden Stichproben hinsichtlich des Nutzungsverhaltens und der Nutzungsintention der fünf Bereiche digitalen Coachings. (Die Kategorie „Digitalisierung des Coachs“ wurde nicht gebildet; das Item ging in die Kategorie „Methoden“ ein. Dafür wurde eine extra Kategorie „Evaluation" gebildet). Ein Vergleich der Mittelwerte zeigt signifikante Unterschiede zwischen externen Coaches und Personalverantwortlichen in der Nutzungsintention bei der Coachwahl $(F(1,191)=11,20 ; p=0,001)$, bei den Methoden $(F(1,191)=26,19 ; p=0,000)$ und den Prozessen $(F(1,191)=11,95 ; p=0,001)$. Bei der tatsächlichen Nutzung gibt es Unterschiede bei der Coachwahl ( $F(1,191)=40,16 ; p=0,000)$ und bei den Methoden $(F(1,191)=5,61 ; p=0,019)$.

Die größten Diskrepanzen zeigen sich bei der Nutzung digitaler Plattformen zur Auswahl von Coaches. Hier haben die Coaches bei der Intention und dem tatsächlichen Nutzungsverhalten deutlich höhere Werte als die Personalverantwortlichen. Die Personalverantwortlichen zeigen zwar auch eine hohe Intention, solche Plattformen zu nutzen, ihr tatsächliches Nutzungsverhalten ist jedoch deutlich geringer ausgeprägt.

Dem Einsatz von digitalen Tools für Coachingmethoden und -prozesse sind die Personalverantwortlichen mehr zugeneigt als die Coaches. Bei der digitalen Evaluation und Kommunikation haben beide Gruppen ähnliche Ausprägungen.

In Abb. 4 und 5 sind zwei Regressionsmodelle dargestellt, welche die Wirkung der Einstellung, der sozialen Norm und der Verhaltenskontrolle auf die Intention und damit auf das tatsächliche Nutzungsverhalten digitaler Plattformen und Tools im Coaching erklären. Die Methode der Regressionsanalyse wurde gewählt, da aufgrund des zu geringen Stichprobenumfangs Pfadanalysen bzw. Strukturgleichungsmodelle für diese Untersuchung nicht geeignet sind.

Betrachtet man zunächst die Korrelationen zwischen den Einflussgrößen auf der linken Seite der Modelle, zeigt sich, dass bei den externen Coaches alle Variablen hoch miteinander korrelieren, während bei den Personalverantwortlichen die Einstellungen relativ unabhängig von der Verhaltenskontrolle sind.

Bei beiden Modellen ist die soziale Norm gegenüber den anderen Einflussgrößen jeweils der stärkste signifikante Prädiktor für die Nutzungsintention. Bei den Personalverantwortlichen sind die Einstellungen ebenfalls ein signifikanter Prädiktor.
Abb. 3 Häufigkeiten bezüglich der Nutzung und Nutzungsintention digitaler Plattformen und Tools für Coaching von externen Coaches $(N=102)$ und Personalverantwortlichen $(N=91)$

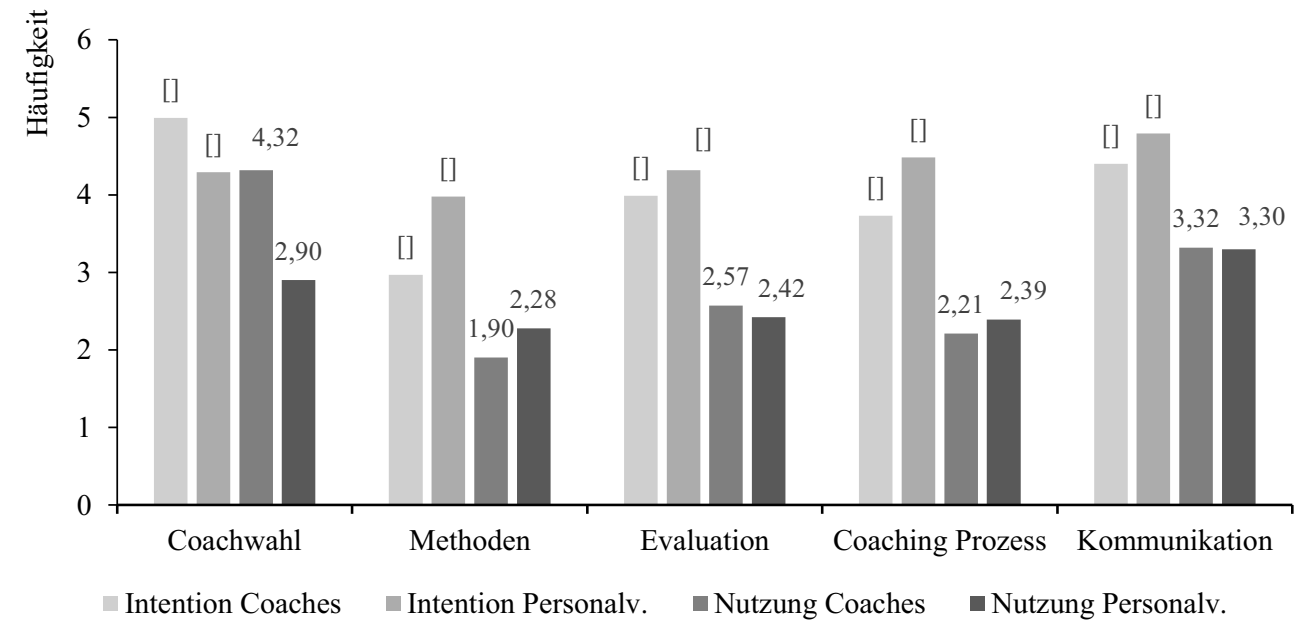




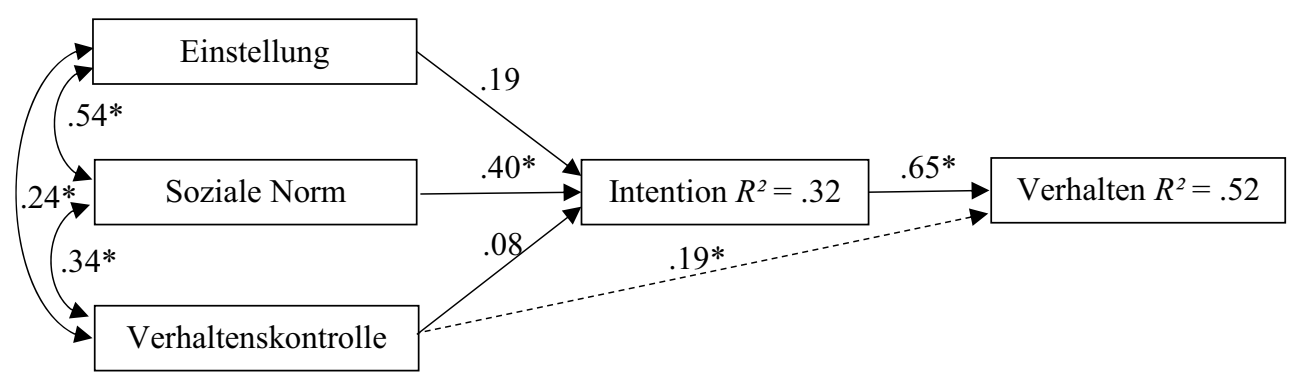

Abb. 4 Regressionsmodelle zur Vorhersage der Nutzung digitaler Plattformen und Tools im Coaching für externe Coaches $(N=102)$. Intention: $(F(3,98)=15,30 ; p=0,000)$ und Verhalten $(F(1,100)=54,16, p=0,000)$ (Signifikante Koeffizienten sind mit * gekennzeichnet, die geraden Pfeile stehen für Regressionsgewichte $(\beta)$, die gekrümmten Doppelpfeile für Korrelationen (r))

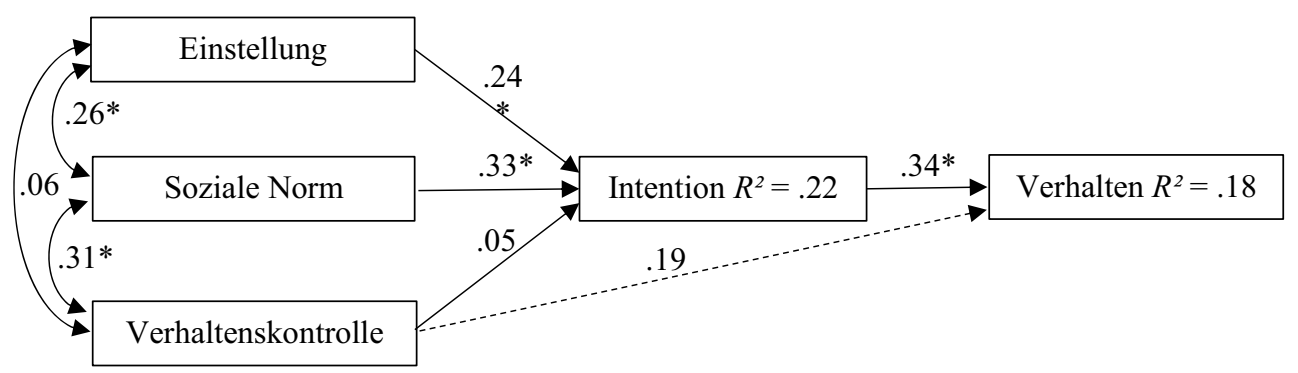

Abb. 5 Regressionsmodelle zur Vorhersage der Nutzung digitaler Plattformen und Tools im Coaching für Personalverantwortliche $(N=91)$. Intention: $(F(3,87)=8,15 ; p=0,000)$ und Verhalten $(F(1,89)=9,32 ; p=0,000)$ (Signifikante Koeffizienten sind mit * gekennzeichnet, die geraden Pfeile stehen für Regressionsgewichte $(\beta)$, die gekrümmten Doppelpfeile für Korrelationen ( $\mathrm{r})$ )

Abb. 6 Einstellung bezüglich der Nutzung digitaler Plattformen und Tools für Coaching von externen Coaches $(N=102)$ und Personalverantwortlichen $(N=91)$

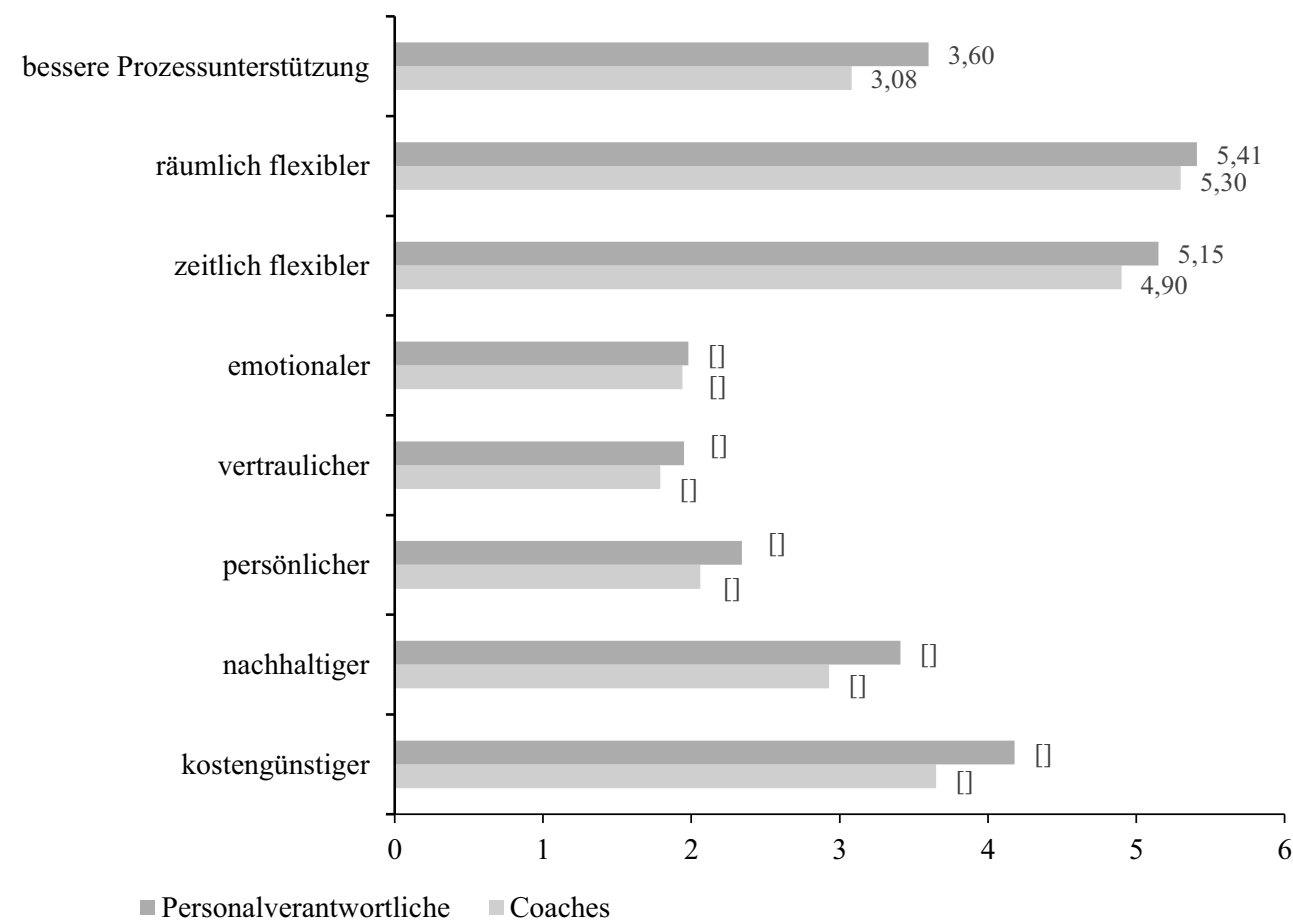

Die Wirkung der Intentionen und der Verhaltenskontrolle auf das Verhalten wurde mit zwei weiteren linearen Regressionen analysiert. Diese zeigen bei den Coaches einen starken Einfluss auf das tatsächliche Verhalten, während bei den Personalverantwortlichen der Zusammenhang zwar noch signifikant, jedoch deutlich schwächer ausfällt. Bei den Coaches erweist sich in dieser Analyse im Gegensatz zu den Personalverantwortlichen die Verhaltenskontrolle als zusätzlicher signifikanter Prädiktor für das Verhalten (im Modell durch eine gestrichelte Linie dargestellt). Insgesamt 
ist die Varianzaufklärung für das Verhalten bei den Coaches deutlich höher als bei den Personalverantwortlichen.

Betrachten wir abschließend die Einstellungen hinsichtlich der Nutzung von digitalen Plattformen und Tools im Coaching detaillierter. Der Mittelwertvergleich der Facetten der Einstellungen, also auf Itemebene, zeigt, dass die Personalverantwortlichen insgesamt positiver auf digitale Plattformen und Tools blicken, als es die Coaches tun. Signifikante Unterschiede zeigen sich bei den Items ,kostengünstiger" $(F(1,191)=6,68 ; p=0,010)$, ,nachhaltiger" $(F(1,191)=10,86 ; p=0,022)$ und ,bessere Prozessunterstützung" $(F(1,191)=13,30 ; p=0,012)$.

Insgesamt zeigt der detaillierte Blick (siehe Abb. 6), dass bei den Einstellungen zu digitalen Plattformen und Tools im Coaching Effizienzkriterien, also Kostenersparnis sowie zeitliche und räumliche Flexibilität ausschlaggebend sind, jedoch keine Erwartungen, dass sich dadurch die Qualität des Coachings verbessert. Es wird eher eine qualitative Verschlechterung in Kauf genommen, was sich darin zeigt, dass die Beziehungsparameter Vertrauen, Emotionalität und persönliche Nähe im Mittel einen Skalenpunkt unterhalb der Skalenmitte sind.

\section{Diskussion}

Zusammenfassend lässt sich festhalten: Der Vergleich von Personalverantwortlichen in Organisationen und externen Coaches zeigt bezüglich der Nutzung digitaler Tools im Coaching, dass Personalverantwortliche der Digitalisierung im Coaching grundsätzlich aufgeschlossener gegenüberstehen als die Coaches und sich dabei stärker an der sozialen Norm orientieren. Eine mögliche Erklärung hierfür ist deren Unternehmens- bzw. Organisationszugehörigkeit und ein damit verbundener höherer Innovations- und Effizienzdruck als bei externen, selbstständigen Coaches. Im Gegensatz zu den eingangs beschriebene Forschungsergebnissen zur Theory of Planned Action, bei denen die Einstellungen generell der stärkste Prädiktor für die Intention waren (vgl. Ajzen 2005), ist in der vorliegenden Untersuchung die soziale Norm für beide Gruppen ausschlaggebend für die Nutzung digitaler Plattformen und Tools im Coaching. Es geht hier also um ein Verhalten, das nur zu einem kleineren Teil selbst entschieden werden kann, sondern im Wesentlichen durch die Entwicklungen des Marktes hervorgebracht wird.

Hinsichtlich der ersten Forschungsfrage nach der aktuellen bzw. zukünftigen Nutzung digitaler Angebote im Coaching zeigt sich die größte Diskrepanz bei der Nutzung digitaler Plattformen zur Auswahl von Coaches. Hier weisen die Coaches sowohl bei der Intention als auch beim tatsächlichen Nutzungsverhalten deutlich höhere Werte auf als die Personalverantwortlichen. Darin können sich die Vorteile für externe Coaches, auf solchen Plattformen für eine effi- ziente Akquise präsent zu sein, widerspiegeln. Zwar zeigen auch Personalverantwortliche eine hohe Intention, solche Plattformen zu nutzen, ihr tatsächliches Nutzungsverhalten ist jedoch deutlich geringer ausgeprägt. Dies könnte durch organisationsbedingte Restriktionen zu erklären sein. AuBerdem zeigen sich Personalverantwortliche dem Einsatz von digitalen Coachingmethoden sowie digitalisierten Coachingprozessen mehr zugeneigt als externe Coaches. Bei der digitalen Evaluation sowie Kommunikation weisen beide Gruppen ähnliche Ausprägungen auf, hier herrschen also weitgehend ähnliche Vorstellungen über Nutzen und Nutzung bei Personalverantwortlichen und externen Coaches.

Die Ergebnisse zur zweiten Forschungsfrage, inwiefern Einstellungen, subjektive Norm und wahrgenommene Verhaltenskontrolle von externen Coaches und Personalverantwortlichen die Nutzungsintention und das Nutzungsverhalten von digitalen Tools im Coaching beeinflussen, zeigen zunächst, dass bei externen Coaches alle Variablen hoch miteinander korrelieren, hingegen bei den Personalverantwortlichen die Einstellungen relativ unabhängig von der Verhaltenskontrolle sind. Dies könnte darin begründet liegen, dass Personalverantwortliche meist nicht selbst coachen bzw. den Coachingprozess nicht direkt gestalten und dass die Einstellungen sich somit unabhängig von den tatsächlichen Möglichkeiten bzw. Fähigkeiten, das Verhalten auch zu zeigen, ausprägen. Weiterhin ist die soziale Norm bei beiden Berufsgruppen gegenüber den anderen Einflussgrößen jeweils der stärkste und einzig signifikante Prädiktor für die Nutzungsintention. Die Wirkung der Intention auf das Verhalten weist bei externen Coaches einen starken Einfluss auf das tatsächliche Verhalten auf. Bei den Personalverantwortlichen ist der Zusammenhang zwar auch noch signifikant, fällt jedoch deutlich schwächer aus. Die lässt sich wiederum durch die weniger konkreten Nutzungsmöglichkeiten digitaler Plattformen und Tools im Coaching durch die Personalverantwortlichen begründen, möglicherweise bedingt durch organisationale Restriktionen oder einfach nur dadurch, dass sie zwar durch ihre Rolle in der Organisation mit Coaching zu tun haben, aber nicht selbst coachen.

Schließlich zeigen die Ergebnisse zur Beantwortung der dritten Forschungsfrage, welche Unterschiede es zwischen Einstellungen, subjektiver Norm und wahrgenommener Verhaltenskontrolle sowie Intention und Verhalten zwischen externen Coaches und Personalverantwortlichen bezüglich der Nutzung digitaler Plattformen und Tools im Coaching gibt, dass Personalverantwortliche insgesamt positiver auf digitale Plattformen und Tools blicken als externe Coaches. Dies spiegelt sich vor allem in höheren Einstellungsausprägungen der Personalverantwortlichen hinsichtlich Kostenersparnissen, Nachhaltigkeit und besserer Prozessunterstützung durch digitalisiertes Coaching wider. Für die Einstellungen von Personalverantwortlichen und auch die von 
Coaches zum Einsatz digitaler Plattformen und Tools im Coaching sind also Effizienzkriterien wie Kostenersparnisse sowie zeitliche und räumliche Flexibilität ausschlaggebend. Gleichzeitig hegen beide Berufsgruppen jedoch keine Erwartungen, dass sich durch die Digitalisierung die Qualität des Coachings verbessert, es wird im Gegenteil in Kauf genommen, dass für wesentliche Parameter, nämlich hinsichtlich der Beziehungsqualität in Bezug auf Nähe, Vertrauen und Emotionalität, sich Nachteile ergeben werden. Dies ist insofern bedenkenswert, als dass in zahlreichen Studien die Beziehung im Coaching als wichtigster Prädiktor für die Wirksamkeit beschrieben und ermittelt wird (vgl. z. B. Jansen et al. 2004; Ianiro et al. 2013).

Die vorliegende Untersuchung weist einige methodische Limitationen auf. Da es sich bei den Stichproben um convenience samples, also nicht-repräsentative, anfallende Stichproben handelt, können die Ergebnisse nur bedingt generalisiert werden. Da die Teilnehmenden z. B. ausschließlich Personalverantwortliche und Coaches aus dem deutschsprachigen Raum sind, können die Ergebnisse nicht auf andere Nationalitäten bzw. Kulturen übertragen werden. Hinzu kommt, dass der Großteil der Coaches überwiegend nicht für Selbstzahler arbeitet. Dies könnte einen elementaren Einflussfaktor hinsichtlich der subjektiven Norm darstellen. Weiterhin eruiert der Fragebogen das individuelle Nutzungsverhalten von Personalverantwortlichen, wobei in größeren Unternehmen die Nutzungsmöglichkeiten oftmals vorgegeben werden. Bachmann (2016) spricht in diesem Kontext davon, dass die Definitionsmacht von Coaching letztlich bei den Organisationen selbst liegt. So werden Coaches beispielsweise vielfach über Rahmenvertragsausschreibungen speziell für den Einsatz im Unternehmen und nicht über Bewertungen auf Online-Plattformen ausgewählt. Oder die zur Verfügung stehenden Coaching-Formate sind vom Unternehmen vorgegeben und für Personalverantwortliche nicht frei wählbar. Insofern ist es denkbar, dass die Teilnehmenden zwar eine hohe Nutzungsintention aufweisen, diese jedoch in ihrer Umsetzung im Praxisalltag durch die Rahmenbedingungen von Organisationen beschränkt werden. Ebenso können diese organisationalen Rahmenbedingungen auch das tatsächliche Nutzungsverhalten digitalisierten Coachings von Personalverantwortlichen negativ beeinflussen. Dies kann ein weiterer Erklärungsgrund für das im Vergleich zur Intention signifikant niedriger ausfallende Nutzungsverhalten sein. Außerdem impliziert die Wahl der Theorie des geplanten Verhaltens (Ajzen 1985; 1991) als theoretische Grundlage der Studie eine geringe Generalisierbarkeit auf andere Arbeitsbereiche oder Berufsgruppen. Alternative Modelle wie das Technology Acceptance Model (Davis und Venkatesh 1996) hätten möglicherweise technik-spezifischere Aspekte des Nutzungsverhaltens digitalisierten Coachings von Personalverantwortlichen und Coaches wie wahrgenommene Nützlich- keit und wahrgenommene Nutzerfreundlichkeit beleuchtet, wobei die wahrgenommene Nützlichkeit in der vorliegenden Untersuchung im Rahmen der Einstellung mitabgedeckt wurde.

Prägnant zusammengefasst kann man sagen, dass externe Coaches, die häufiger Karriere- und Work-Life-Balance-Themen bearbeiten, als es bei Organisationscoachings der Fall ist, eher noch zurückhaltend mit digitalen Plattformen und Tools im Coaching umgehen, wenn man von der Plattformnutzung zur Selbstpräsentation und zum Gefundenwerden einmal absieht. Diese Nutzungsform hat sich allerdings schon seit den Anfang 2000er Jahren etabliert, etwa im deutschsprachigen Raum mit der Coach-Datenbank von Rauen. Weiterhin sind bei den externen Coaches die Einstellungen zu digitalen Angeboten im Coaching stark mit der sozialen Norm und der Verhaltenskontrolle, also mit den technischen Möglichkeiten und Fähigkeiten korreliert. Dieses Kontrollerleben könnte auch als Technikaffinität interpretiert werden. Die Nutzungsintention wird fast ausschließlich durch die soziale Norm prädiktiert, das Verhalten durch die Intention und die wahrgenommene Kontrolle. Bei der Nutzung digitaler Plattformen und Tools im Coaching beugen sich die befragten Coaches also dem sozialen Druck und nehmen dabei Verschlechterungen des Coachings bezogen auf die Beziehungsqualität in Kauf bei gleichzeitigen Vorteilen der räumlich-zeitlichen Flexibilität sowie aus Opportunitätsgründen bezüglich der Entwicklungen im Coachingmarkt. Die letztendliche Nutzung wird weiterhin noch durch die Technikaffinität prädiktiert.

Für die Personalverantwortlichen lässt sich zusammenfassen, dass diese insgesamt eine höhere Nutzungsintention für digitale Plattformen und Tools angeben, die tatsächliche Nutzung in den Organisationen jedoch dahinter zurückbleibt. Dies trifft erstaunlicherweise auch auf die Plattformen zu, auf denen Coaches sich präsentieren und gefunden werden können. Hier scheinen für die Personalverantwortlichen persönliche Netzwerke, Empfehlungen bzw. digitale soziale Netzwerke wie xing.com oder linked.com eine wichtigere Rolle zu spielen. Die Einstellungen, soziale Norm und Verhaltenskontrolle sind bei den Personalverantwortlichen unabhängiger voneinander. Sie stehen digitalen Plattformen und Tools im Coaching positiver gegenüber, beugen sich aber genauso dem sozialen Druck. Die subjektive Verhaltenskontrolle spielt bei ihnen keine Rolle. Das liegt möglicherweise daran, dass sie das Thema eher aus einer weniger involvierten und identifizierten Perspektive betrachten. Effizienz und Flexibilität stehen im Vordergrund, es geht wohl eher darum, als interner Dienstleister nützlich zu sein, denn das eigentliche Coaching wird ja - technisch und persönlich - von den Coaches erbracht, als deren Auftraggebende sie agieren. 
Open Access Dieser Artikel wird unter der Creative Commons Namensnennung 4.0 International Lizenz (http://creativecommons.org/ licenses/by/4.0/deed.de) veröffentlicht, welche die Nutzung, Vervielfältigung, Bearbeitung, Verbreitung und Wiedergabe in jeglichem Medium und Format erlaubt, sofern Sie den/die ursprünglichen Autor(en) und die Quelle ordnungsgemäß nennen, einen Link zur Creative Commons Lizenz beifügen und angeben, ob Änderungen vorgenommen wurden.

\title{
Anhang
}

Tab. 4 Verwendete Items im Fragebogen

\author{
Skala Einstellung \\ Durch die Digitalisierung ... \\ Im Coaching können sich zukünftig Kostenersparnisse ergeben \\ Kann Coaching nachhaltiger werden \\ Kann Coaching zukünftig persönlicher werden \\ Kann Coaching zukünftig weniger vertraulicher werden \\ Kann Coaching zukünftig emotionaler werden \\ Kann Coaching zukünftig zeitlich flexibler werden \\ Kann Coaching zukünftig räumlich flexibler werden \\ Im Coaching lassen sich neue Erkenntnisse u. Reflexionsprozesse besser unterstützen
}

\section{Skala Subjektive Norm}

Befreundete Coaches setzten immer mehr digitale Medien, Apps und Plattformen im Coaching ein Unter Coaches ist es mir wichtig auf dem neuesten Stand zu sein

Positive Erfahrungen anderer Coaches mit digitalen Medien, Apps und Plattformen im Coaching lösen bei mir Interesse und Neugier aus Ich bemerke, dass immer mehr Klienten/innen den Einsatz digitaler Medien, Apps und Plattformen im Coaching fordern Auch andere Coaches legen Wert auf den Einsatz digitaler Medien im Coaching

Ich denke, dass sich auch Coaching dieser gesellschaftl. Entwicklung auf Dauer nicht entziehen kann

\section{Skala Wahrgenommene Verhaltenskontrolle}

Ich habe ausreichend finanz. Ressourcen, um digitale Coachingformate zu verwenden

Ich habe genüg. Zeit, um mich mit den digit. Möglichkeiten f. Coaching auseinanderzusetzen

Ich weiß, wie ich digitale Coachingformate implementieren kann

Es fällt mir leicht immer auf dem neusten Stand der Technik zu bleiben

Mir fällt es leicht mit digitalen Geräten oder Plattformen umzugehen

Probleme bei der Nutzung digitaler Geräte u. Apps kann ich selbst schnell beheben

\section{Skala Intention/Verhalten}

Für den Einsatz von Coaching in meiner Organisation bzw. meinem Unternehmen nutze ich bereits/kann ich mir zukünftig vorstellen folgende digitale Angebote zu nutzen ...

Online-Plattformen, um Coaches zu finden

Online-Plattformen, um Coaches mit einem bestimmten Qualitäts- und Professionsstandard zu finden

Online-Plattformen, um Coaches zu bewerten

Online-Plattformen, um Coaches entsprechend ihrer Bewertungen auswählen zu können

Online-Tools zur Unterstützung des Coachings durch Angebotsstellung, Terminplanung und Rechnungslegung

Online-Tools zur Unterstützung des Coachings durch Dokumentation, Visualisierungen oder Hausaufgaben

Online-Tools zur Unterstützung des Coachings durch Erinnerungen oder Trackingfunktionen

Online-Tools zur Unterstützung des Coachings durch Evaluation und Feedback an/über den Coach

Digitale Methoden bzw. Tools (z. B. digitales Whiteboard, Fragebögen, Aufstellungsfiguren, Bilder usw.)

Online-Kommunikationskanäle (z. B. Chat, Audio, Video)

Selbstcoaching-Tools, die die Arbeit von Coaches teilweise oder ganz ersetzen (z. B. Selbstcoaching in Form von Texten, Videos, digital angeleiteten Modulen usw.)

Coaching in virtuellen Räumen oder Realitäten, vermittelt durch Avatare 


\section{Literatur}

Ajzen, I. (1985). From intentions to actions: a theory of planned behavior. In J. Kuhl \& J. Beckmann (Hrsg.), Action-control: from cognition to behavior (S. 11-39). Heidelberg: Springer.

Ajzen, I. (1991). The theory of planned behavior. Organizational Behavior and Human decision processes, 50, 179-211.

Ajzen, I. (2005). Attitudes, personality, and behavior. Berkshire: McGraw-Hill.

Armitage, C. J., \& Conner, M. (2001). Efficacy of the theory of planned behaviour: a meta-analytic review. British journal of social psychology, 40(4), 471-499.

Bachmann, T. (2016). Implizite Theorien über Lernen und Veränderungen durch Coaching in Organisationen - Eine empirische Untersuchung. Organisationsberatung, Supervision, Coaching, 23(3), 231-253.

Bachmann, T., \& Fietze, B. (2018). Die Digitalisierung von Coaching - Gedanken aus der Perspektive teilnehmender Beobachtung. Organisationsberatung. Supervision, Coaching, 25(3), 281-292.

Bortz, J. (2013). Lehrbuch der Statistik: Für Sozialwissenschaftler. Berlin, Heidelberg: Springer.

Carter, A. (2006). Practical methods for evaluating coaching. reportinstitute for employment studies, Bd. 430.

Davis, F. D., \& Venkatesh, V. (1996). A critical assessment of potential measurement biases in the technology acceptance model: three experiments. International Journal of Human-Computer Studies, $45,19-45$.

Deutscher Bundesverband Coaching e.V. (2013). Coaching Marktanalyse 2013. https://coach-trainer-akademie.ch/fileadmin/user_ upload/pdf/Coaching_Studie_Uni_Marburg_2013.pdf, Zugegriffen: 26.10.2018.

Diekmann, A. (2017). Empirische Sozialforschung. Grundlagen, Methoden, Anwendungen (11. Aufl.). Reinbek: Rowohlt.

Fietze, B. (2016). Grenzenlose Beratung? Die Digitalisierung rüttelt an den Grundfesten. Journal bso, 3, 6-10.
Geißler, H. (2018). E-Coaching—State of the art 2016. In J. Heller, C. Triebel, B. Hauser \& A. Koch (Hrsg.), Digitale Medien im Coaching (S. 15-31). Berlin, Heidelberg: Springer.

Hausenblas, H. A., Carron, A. V., \& Mack, D. E. (1997). Application of the theories of reasoned action and planned behavior to exercise behavior: A meta-analysis. Journal of Sport and Exercise Psycho$\log y, 19,36-51$.

Heller, J., Triebel, C., Hauser, B., \& Koch, A. (2018). Digitale Medien im Coaching. Berlin, Heidelberg: Springer.

Ianiro, P. M., Schermuly, C.C., \& Kauffeld, S. (2013). Why interpersonal dominance and affiliation matter: an interaction analysis of the coach-client relationship. Coaching: An International Journal of Theory, Research and Practice, 6(1), 25-46.

Jansen, A., Mäthner, E., \& Bachmann, T. (2004). Erfolgreiches Coaching: Wirkfaktoren im Einzel-Coaching. Kröning: Asanger.

Pascal, A., Sass, M., \& Gregory, J. B. (2015). I'm only human: The role of technology in coaching. Consulting Psychology Journal: Practice and Research, 67(2), 100.

Randall, D. M., \& Wolff, J.A. (1994). The time interval in the intentionbehaviour relationship: Meta-analysis. British Journal of Social Psychology, 33, 405-418.

Rauen, C. (2003). Coaching. Göttingen: Hogrefe.

Schermuly, C. C., Schröder, T., Nachtwei, J., Kauffeld, S., \& Gläs, K. (2012). Die Zukunft der Personalentwicklung. Zeitschrift Für Arbeits- und Organisationspsychologie A\&O, 56(3), 111-122.

Schwaemmle, U., \& Staehelin, P. (2002). Rolle, Perspektiven und Arbeitsweisen von internen und externen Coaches. In W. Vogelauer (Hrsg.), Coaching Praxis (S. 109-123). München: Luchterhand.

Wiethoff, C. (2004). Motivation to learn and diversity training: application of the theory of planned behavior. Human Resource Development Quarterly, 15(3), 263-278.

Publisher's Note Springer Nature remains neutral with regard to jurisdictional claims in published maps and institutional affiliations. 